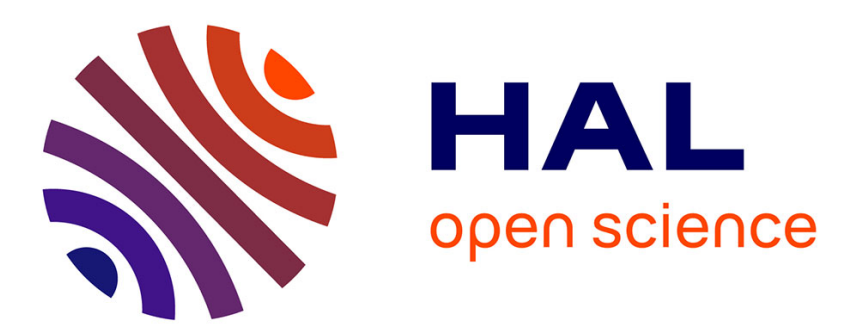

\title{
Contribution à l'étude du pompage optique par échange de métastabilité dans 3He. - Deuxième Partie
}

\author{
J. Dupont-Roc, M. Leduc, F. Laloë
}

\section{To cite this version:}

J. Dupont-Roc, M. Leduc, F. Laloë. Contribution à l'étude du pompage optique par échange de métastabilité dans 3He. - Deuxième Partie. Journal de Physique, 1973, 34 (11-12), pp.977-987. 10.1051/jphys:019730034011-12097700 . jpa-00208120

\section{HAL Id: jpa-00208120 https://hal.science/jpa-00208120}

Submitted on 1 Jan 1973

HAL is a multi-disciplinary open access archive for the deposit and dissemination of scientific research documents, whether they are published or not. The documents may come from teaching and research institutions in France or abroad, or from public or private research centers.
L'archive ouverte pluridisciplinaire HAL, est destinée au dépôt et à la diffusion de documents scientifiques de niveau recherche, publiés ou non, émanant des établissements d'enseignement et de recherche français ou étrangers, des laboratoires publics ou privés. 


\footnotetext{
Classification

Physics Abstracts

13.20
}

\title{
CONTRIBUTION A L'ÉTUDE DU POMPAGE OPTIQUE PAR ÉCHANGE DE MÉTASTABILITÉ DANS ${ }^{3} \mathrm{He}$
}

\author{
Deuxième Partie $(*)$ \\ J. DUPONT-ROC, M. LEDUC et F. LALÖ̈ \\ Laboratoire de Spectroscopie Hertzienne de l'ENS \\ 24, rue Lhomond, 75005 Paris, France
}

(Reçu le 14 juin 1973)

\begin{abstract}
Résumé. - Dans ce second article, nous nous intéressons aux expériences de résonance magnétique sur des atomes de ${ }^{3} \mathrm{He}$ squmis au pompage optique « indirect " par l'intermédiaire du niveau métastable $2{ }^{3} \mathrm{~S}_{1}$. On calcule les caractéristiques des résonances dans le niveau fondamental et dans les deux sous-niveaux hyperfins du métastable $2{ }^{3} \mathrm{~S}_{1}$. On utilise pour cela les résultats établis dans le premier article et relatifs aux collisions d'échange de métastabilité ; celles-ci introduisent en effet d'importants couplages entre les orientations des différents niveaux étudiés. On présente ensuite brièvement les expériences effectuées pour vérifier certains points de la théorie précédente, relatives aux largeurs, intensités et positions des courbes de résonance magnétique observées tant pour l'état fondamental que métastable.
\end{abstract}

\begin{abstract}
We are mainly concerned here with magnetic resonance experiments with ${ }^{3} \mathrm{He}$ atoms undergoing indirect optical pumping via the $2{ }^{3} \mathrm{~S}_{1}$ metastable state. The characteristics of the resonances in the ground state and in the two hyperfine sublevels of the $2{ }^{3} \mathrm{~S}_{1}$ metastable state are calculated. Use is made of the results of the previous paper about metastability exchange collisions which produce strong coupling between the orientations of the different atomic levels under study. We briefly report experiments which were performed in order to check several aspects of the previous theory. They deal with the widths, intensities and positions of the magnetic resonance curves observed in the ground state and in the $2{ }^{3} \mathrm{~S}_{1}$ metastable hfs sublevels.
\end{abstract}

Introduction. - Dans la première partie de cet article publié dans ce même numéro [1], nous avons étudié de façon générale la cinétique du pompage optique de ${ }^{3} \mathrm{He}$ en présence d'un champ magnétique statique $\mathbf{B}_{0}$. En pratique, de nombreuses expériences utilisent également un champ de radiofréquence $\mathbf{B}_{1}(t)$, permettant d'effectuer la résonance magnétique. L'objet de cette seconde partie est d'étudier en détail de telles expériences sur le plan théorique. Des vérifications expérimentales sont également présentées.

4. Résonance magnétique dans le niveau fondamental ou métastable. - Le calcul des valeurs stationnaires des diverses composantes de l'orientation d'un niveau dans lequel on effectue la résonance magnétique est bien connu (équations de Bloch); on pourrait donc penser a priori qu'il suffit d'appliquer les résultats de ce calcul aux orientations soit du niveau fonda-

$\left(^{*}\right)$ La première partie de cet article est publiée dans le même numéro du J. Physique 34 (1973) 961. mental, soit du niveau $F=\frac{1}{2}$, soit du niveau $F=\frac{3}{2}$, suivant que la fréquence du champ oscillant est proche de la fréquence de résonance de l'un de ces trois niveaux. En fait, ce n'est pas possible, car le calcul classique suppose que le niveau étudié est isolé (c'est-à-dire que ses observables ne sont couplées à celles d'aucun autre niveau), condition qui n'est pas réalisée dans le pompage optique de l'hélium; comme nous l'avons vu dans les paragraphes 2 et 3 précédents, même si le champ $\mathbf{B}_{0}$ est assez élevé pour rendre non séculaires les couplages entre orientations transversales, les couplages entre orientations longitudinales (de fréquences propres nulles) sont toujours séculaires et jouent de ce fait un rôle essentiel. Il est donc nécessaire de généraliser les calculs classiques pour traiter le cas où la résonance magnétique concerne à la fois plusieurs niveaux; c'est ce que nous allons faire dans ce paragraphe 4 .

4. 1 Position DU PROBlÈME. - Nous supposons ici que le champ $\mathbf{B}_{1}(t)$ est perpendiculaire à $\mathbf{B}_{0}$ et tourne autour de $\mathbf{B}_{0}$ avec la vitesse angulaire $\omega$. 
Comme pour $\mathbf{B}_{0}$, caractérisons l'intensité du champ $B_{1}$ par les pulsations :

$$
\left\{\begin{array}{l}
\omega_{1}=-\gamma_{\mathrm{m}} B_{1} \\
\Omega_{1}=-\gamma_{\mathrm{f}} B_{1} .
\end{array}\right.
$$

Pour écrire l'évolution des orientations sous l'effet de $\mathbf{B}_{1}$ passons, suivant la méthode habituelle, dans le « référentiel tournant» $O X Y Z$ en posant :

$$
\left\{\begin{array}{l}
\left\langle F_{+}\right\rangle_{3 / 2}=j_{3 / 2}^{+} \mathrm{e}^{i \omega \mathrm{t}} \\
\left\langle F_{+}\right\rangle_{1 / 2}=j_{1 / 2}^{+} \mathrm{e}^{i \omega \mathrm{t}} \\
\left\langle I_{+}\right\rangle_{\mathrm{f}}=j_{\mathrm{f}}^{+} \mathrm{e}^{i \omega \mathrm{t}} .
\end{array}\right.
$$

Dans ce référentiel, $\mathbf{B}_{1}$ est un champ constant (que nous supposons parallèle à l'axe $O Y$ qui, à $t=0$, coïncide avec $O y$ ) et les équations d'évolution des observables en présence de $\mathbf{B}_{1}$ sont :

$$
\begin{gathered}
\left\{\begin{array}{l}
\frac{\mathrm{d}}{\mathrm{d} t}\left\langle F_{z}\right\rangle_{3 / 2}=-\frac{2 \omega_{1}}{3} \operatorname{Re}_{3 / 2}^{+} \\
\frac{\mathrm{d}}{\mathrm{d} t}\left\langle F_{z}\right\rangle_{1 / 2}=-\frac{4 \omega_{1}}{3} \operatorname{Re}_{j}^{+} \\
\frac{\mathrm{d}}{\mathrm{d} t}\left\langle I_{z}\right\rangle_{\mathrm{f}}=-\Omega_{1} \operatorname{Re} j_{\mathrm{f}}^{+}
\end{array}\right. \\
\left\{\begin{array}{l}
\frac{\mathrm{d}}{\mathrm{d} t} j_{3 / 2}^{+}=i\left[\frac{2 \omega_{\mathrm{m}}}{3}-\omega\right] j_{3 / 2}^{+}+\frac{2 \omega_{1}}{3}\left\langle F_{z}\right\rangle_{3 / 2} \\
\frac{\mathrm{d}}{\mathrm{d} t} j_{1 / 2}^{+}=i\left[\frac{4 \omega_{\mathrm{m}}}{3}-\omega\right] j_{1 / 2}^{+}+\frac{4 \omega_{1}}{3}\left\langle F_{z}\right\rangle_{1 / 2} \\
\frac{\mathrm{d}}{\mathrm{d} t} j_{\mathrm{f}}^{+}=i\left[\omega_{\mathrm{f}}-\omega\right] j_{\mathrm{f}}^{+}+\Omega_{1}\left\langle I_{z}\right\rangle_{\mathrm{f}} .
\end{array}\right.
\end{gathered}
$$

Ajoutons ces termes aux seconds membres des éq. (1.46a) et (1.46b), après y avoir effectué la substitution (4.2); nous obtenons alors un système de 6 équations couplées donnant l'évolution de $j_{3 / 2}^{+}$, $j_{1 / 2}^{+}, j_{\mathrm{f}}^{+},\left\langle F_{z}\right\rangle_{3 / 2},\left\langle F_{z}\right\rangle_{1 / 2},\left\langle I_{z}\right\rangle_{\mathrm{f}}$. Du fait de la présence au second membre de $(4.3 a)$ de parties réelles $\left(\operatorname{Re} j_{3 / 2}^{+}=\frac{1}{2} j_{3 / 2}^{+}+\frac{1}{2}\left(j_{3 / 2}^{+}\right)^{*}\right)$, ce système est en réalité un système de 9 équations linéaires couplées, à coefficients constants, entre $j_{3 / 2}^{+},\left(j_{3 / 2}^{+}\right)^{*},\left\langle F_{z}\right\rangle_{3 / 2}$, etc..

Nous voyons donc que la présence du champ $\mathbf{B}_{1}$, qui couple trois groupes d'équations auparavant indépendants, complique nettement le problème. Un certain nombre de résultats obtenus plus haut restent toutefois encore valables. Par exemple, nous savons par continuité que les valeurs propres des différents modes tendent, lorsque $B_{1} \rightarrow 0$, vers celles que nous avons déjà calculées aux paragraphes 2 et 3 ; par suite, il y a 6 modes rapides fortement amortis (dont les constantes de temps sont de l'ordre de $\tau$ ) et 3 modes lents. Comme plus haut, nous nous intéresserons surtout à ces modes lents, et en particulier à celui dont la constante de temps $T_{1}^{*}$ tend vers $T_{1}$ lorsque $B_{1} \rightarrow 0$, c'est-à-dire le mode dont la valeur propre est réelle $\left({ }^{7}\right)$.

Le calcul que nous allons faire est en tous points semblable à celui qui a été mené dans les paragraphes 2 et 3 : nous commencerons par calculer, à l'ordre zéro en $\tau / T, \tau / T_{\mathrm{r}}$, les orientations $\langle\mathbf{F}\rangle_{3 / 2}$ et $\langle\mathbf{F}\rangle_{1 / 2}$ correspondant à une orientation nucléaire donnée; puis, en reportant ces valeurs dans le second membre des 3 équations donnant l'évolution de $\langle\mathbf{I}\rangle_{\mathrm{f}}$, nous obtiendrons cette évolution au premier ordre en $\tau / T, \tau / T_{\mathrm{r}}$.

Dans un premier temps, nous devons donc résoudre un système de 6 équations linéaires algébriques donnant les valeurs de $\langle\mathbf{F}\rangle_{3 / 2}$ et $\langle\mathbf{F}\rangle_{1 / 2}$ en fonction de $\langle\mathbf{I}\rangle_{\mathrm{f}}$, dans un mode lent. Bien que sans difficulté de principe, un tel calcul conduit à des expressions peu maniables, et c'est pourquoi nous allons faire des hypothèses simplificatrices supplémentaires. Pour étudier la résonance magnétique sur le niveau fondamental, nous supposerons que le champ $\mathbf{B}_{1}$ est suffisamment faible pour que son action dans le niveau métastable soit négligeable. Pour la résonance sur les sous-niveaux métastables, nous commencerons par supposer que le champ $\mathbf{B}_{0}$ est intense et interdit toute circulation de cohérence; nous discuterons ensuite rapidement quels peuvent être les effets d'une telle circulation.

4.2 RÉSONANCE DANS LE NIVEAU FONDAMENTAL. Dans une expérience de résonance magnétique sur le niveau fondamental, on utilise des champs $\mathbf{B}_{1}$ faibles (typiquement de quelques milligauss). Pour observer cette résonance, il suffit en effet que

$$
\Omega_{1} \sqrt{T_{1} T_{2}} \simeq 1
$$

[où $T_{1}$ et $T_{2}$ sont les temps de relaxation longitudinal et transversal donnés par $(2.14)$ et $(3.8)$, et qui sont respectivement de l'ordre de grandeur de 10 et $\frac{1}{10}$ seconde]. Dans ces conditions, l'ordre de grandeur de $\omega_{1} \tau$ est $10^{-2}$ ou $10^{-3}$ et on comprend que l'action $\mathrm{du}$ champ de radiofréquence oscillant sur le niveau métastable soit négligeable (nous préciserons dans le paragraphe 4.3 suivant quel doit être l'ordre de grandeur de $B_{1}$ pour que son action dans le niveau métastable soit sensible).

Dans ces conditions, les équations d'évolution des orientations $\langle\mathbf{F}\rangle_{3 / 2}$ et $\langle\mathbf{F}\rangle_{1 / 2}$ données par les deux premières lignes des relations (1.46) restent valables. Le calcul des valeurs stationnaires de ces orientations n'a donc pas à être repris ; c'est celui des paragraphes 2 et 3 précédents, et les formules (2.9) et (3.6) sont directement applicables.

$\left({ }^{7}\right)$ Cette valeur propre reste réelle lorsque $B_{1}$ n'est pas nul En effet, le système linéaire des 9 équations qui donnent les évolutions des composantes sur $O X, O Y$ et $O Z$ des orientations $\langle\mathbf{F}\rangle_{3 / 2},\langle\mathbf{F}\rangle_{1 / 2}$ et $\langle\mathbf{I}\rangle_{\mathrm{f}}$ a des coefficients réels, de sorte que les valeurs propres de la matrice associée sont soit réelles, soit complexes conjuguées deux à deux. Parmi les trois modes lents, il y en a donc nécessairement un qui a une valeur propre réelle.

$\left({ }^{8}\right)$ Cette condition est celle qui, d'après les classiques formules de Bloch, indique la mi-saturation pour un niveau isolé de temps de relaxation $T_{1}$ et $T_{2}$; le fait qu'elle reste valable ici peut être vérifié a posteriori sur la formule (4.5). 
Reportons maintenant ces résultats dans les équations d'évolution de $\langle\mathbf{I}\rangle_{\mathrm{f}}$; il vient :

$$
\begin{aligned}
\frac{\mathrm{d}}{\mathrm{d} t}\left\langle I_{z}\right\rangle_{\mathrm{f}} & =-\frac{1}{T_{1}}\left\langle I_{z}\right\rangle_{\mathrm{f}}-\Omega_{1} \operatorname{Re} j_{\mathrm{f}}^{+}+\frac{I_{0}}{T_{1}} \\
\frac{\mathrm{d}}{\mathrm{d} t} j_{\mathrm{f}}^{+} & =\left[-\frac{1}{T_{2}}+i\left(\omega_{\mathrm{f}}+\delta \omega_{\mathrm{f}}-\omega\right)\right] j_{\mathrm{f}}^{+}+\Omega_{1}\left\langle I_{z}\right\rangle_{\mathrm{f}} .
\end{aligned}
$$

Ces équations généralisent (2.11) et (3.7) pour tenir compte de l'effet du champ $B_{1}$; les constantes $T_{1}$, $T_{2}$ et $\delta \omega_{\mathrm{f}}$ sont définies en (2.14), (3.8) et (3.9). A ce stade, on peut remarquer que le système (4.4) est identique à celui qui donne les variations des composantes de l'orientation d'un niveau isolé sur lequel on effectue la résonance magnétique, de temps de relaxation longitudinal $T_{1}$, transversal $T_{2}$, et de fréquence de résonance $\omega_{\mathrm{f}}+\delta \omega_{\mathrm{f}}$. Nous sommes donc ramenés aux classiques équations de Bloch, et tout se passe comme si le niveau fondamental était isolé, le seul effet de l'échange de métastabilité étant de changer le temps de relaxation $T_{\mathrm{r}}$ en $T_{1}$ pour $\left\langle I_{z}\right\rangle_{\mathrm{f}}$, en $T_{2}$ pour $\left\langle I_{+}\right\rangle_{\mathrm{f}}$, et d'ajouter à $\omega_{\mathrm{f}}$ le déplacement de fréquence $\delta \omega_{\mathrm{f}}$. La valeur stationnaire de $\left\langle I_{z}\right\rangle_{\mathrm{f}}$ est donc donnée par l'expression classique :

$$
\begin{aligned}
\left\langle I_{z}\right\rangle_{\mathrm{f}} & =I_{0} \times \\
& \times\left\{1-\frac{\Omega_{1}^{2} T_{1} / T_{2}}{\left(\omega-\omega_{\mathrm{f}}-\delta \omega_{\mathrm{f}}\right)^{2}+\left(1 / T_{2}\right)^{2}+\Omega_{1}^{2} T_{1} / T_{2}}\right\} .
\end{aligned}
$$

L'effet de la circulation de cohérence sur la courbe de résonance obtenue découle immédiatement de la discussion du paragraphe 3.2 ; cet effet se traduit par un déplacement $\delta \omega_{\mathrm{f}}$ et un affinement de cette résonance [2], [3]. L'expression (3.9) montre que les variations en fonction du champ $B_{0}=-\omega_{\mathrm{m}} / \gamma_{\mathrm{m}} \mathrm{du}$ déplacement sont données par la superposition de deux courbes de dispersion, maximales pour $\omega_{\mathrm{m}} \simeq 1 / \tau$; on a alors $\delta \omega_{\mathrm{f}} \simeq 1 / T$, ce qui montre que l'échange de métastabilité produit un déplacement de la résonance $\mathrm{du}$ fondamental comparable à son élargissement.

4. 3 RÉSONANCE DANS L'UN DES SOUS-NIVEAUX $F=\frac{3}{2}$ ou $F=\frac{1}{2}$. - Pour effectuer une résonance magnétique dans l'un des sous-niveaux $F=\frac{3}{2}$ ou $F=\frac{1}{2}$, il faut un champ $\mathbf{B}_{1}$ plus intense que pour le niveau fondamental et l'action directe de $\mathbf{B}_{1}$ peut être sensible à la fois sur $\langle\mathbf{F}\rangle_{3 / 2}$ et $\langle\mathbf{F}\rangle_{1 / 2}$. Pour éviter des calculs compliqués, nous commencerons par un cas simple, celui où le champ magnétique $\mathbf{B}_{0}$ est assez intense pour que la circulation de cohérence soit négligeable.

4.3.1 Cas où la circulation de cohérence est négligeable. - $\alpha$ ) Calcul de l'évolution de $\left\langle I_{z}\right\rangle_{\mathrm{f}}$. - Supposons donc comme dans le paragraphe $3.1 .1 \alpha$ ) que :

$$
\omega_{\mathrm{m}} \tau \gg 1
$$

condition qui exprime que les différences de fréquences d'évolution des orientations transversales sont grandes devant leur amortissement ((4.6) entraîne que $\omega_{\mathrm{f}} T \gg 1$ puisque $\left.\omega_{\mathrm{f}} \simeq 10^{-3} \omega_{\mathrm{m}}, T \simeq 10^{6} \tau\right)$. En pratique, l'inégalité (4.6) est presque toujours réalisée car elle exprime simplement que l'on choisit une situation où les courbes de résonance magnétique des différents niveaux sont bien séparées. Nous supposerons de plus que :

$$
\omega_{1} \ll \omega_{\mathrm{m}}
$$

(cette condition découle en fait de (4.6) puisque les champs $\mathbf{B}_{1}$ utilisés sont tels que $\omega_{1} \lesssim 1 / \tau$; elle interdit à $\mathbf{B}_{1}$ d'être assez intense pour rendre possible, par sa présence, la circulation de cohérence). En négligeant alors les termes non séculaires de couplage entre $j_{3 / 2}^{+}$et $j_{1 / 2}^{+}$, on obtient :

$$
\left\{\begin{array}{r}
\frac{\mathrm{d}}{\mathrm{d} t} j_{3 / 2}^{+} \simeq-\left[\frac{4}{9 \tau}+\frac{1}{\tau_{\mathrm{r}}}+\frac{1}{\tau_{\mathrm{p}}}+i\left(\omega-\frac{2}{3} \omega_{\mathrm{m}}\right)\right] j_{3 / 2}^{+}+ \\
+\frac{2 \omega_{1}}{3}\left\langle F_{z}\right\rangle_{3 / 2}, \\
\frac{\mathrm{d}}{\mathrm{d} t} j_{1 / 2}^{+} \simeq-\left[\frac{7}{9 \tau}+\frac{1}{\tau_{\mathrm{r}}}+\frac{1}{\tau_{\mathrm{p}}}+i\left(\omega-\frac{4}{3} \omega_{\mathrm{m}}\right)\right] j_{1 / 2}^{+}+ \\
+\frac{4 \omega_{1}}{3}\left\langle F_{z}\right\rangle_{1 / 2} .
\end{array}\right.
$$

Ces équations permettent immédiatement d'exprimer les valeurs stationnaires de $j_{3 / 2}^{+}$et $j_{1 / 2}^{+}$en fonction de celles de $\left\langle F_{z}\right\rangle_{3 / 2}$ et $\left\langle F_{z}\right\rangle_{1 / 2}$ :

$$
\begin{aligned}
& j_{3 / 2}^{+} \simeq \frac{2 \omega_{1} / 3}{\frac{4}{9 \tau}+\frac{1}{\tau_{\mathrm{p}}}+\frac{1}{\tau_{\mathrm{r}}}+i\left(\omega-\frac{2 \omega_{\mathrm{m}}}{3}\right)}\left\langle F_{z}\right\rangle_{3 / 2} \\
& j_{1 / 2}^{+} \simeq \frac{4 \omega_{1} / 3}{\frac{7}{9 \tau}+\frac{1}{\tau_{\mathrm{p}}^{\prime}}+\frac{1}{\tau_{\mathrm{r}}}+i\left(\omega-\frac{4 \omega_{\mathrm{m}}}{3}\right)}\left\langle F_{z}\right\rangle_{1 / 2} .
\end{aligned}
$$

Repörtons alors ces résultats dans le second membre des éq. (4.3a); nous obtenons ainsi l'évolution de $\left\langle F_{z}\right\rangle_{3 / 2}$ et $\left\langle F_{z}\right\rangle_{1 / 2}$ due à la radiofréquence et, en ajoutant cette évolution au second membre de (1.46a), leur évolution globale. On remarque aisément que les équations ainsi obtenues sont celles du paragraphe 2 , à condition d'y remplacer $1 / \tau_{\mathrm{p}}$ et $1 / \tau_{\mathrm{p}}^{\prime} \operatorname{par}\left({ }^{9}\right)$ :

$$
\left\{\begin{array}{l}
\frac{1}{\tau_{\mathrm{p}}^{*}}=\frac{1}{\tau_{\mathrm{p}}}+\frac{1}{\tau} F\left(\omega_{1}\right) \\
\frac{1}{\tau_{\mathrm{p}}^{\prime *}}=\frac{1}{\tau_{\mathrm{p}}^{\prime}}+\frac{1}{\tau} F^{\prime}\left(\omega_{1}\right)
\end{array}\right.
$$

( $\left.{ }^{9}\right)$ La substitution (4.10) doit être effectuée sans changer les termes source en $\Phi / \tau_{\mathbf{p}}, \Phi^{\prime} / \tau_{\mathbf{p}}^{\prime}$ qui figurent au second membre dans $\frac{\mathrm{d}}{\mathrm{d} t}\left\langle F_{z}\right\rangle_{3 / 2}, \frac{\mathrm{d}}{\mathrm{d} t}\left\langle F_{z}\right\rangle_{1 / 2}$. 
avec :

$\left\{\begin{array}{c}F\left(\omega_{1}\right)=\left(\frac{2 \omega_{1}}{3}\right)^{2} \frac{\frac{4}{9}+\frac{\tau}{\tau_{\mathrm{p}}}+\frac{\tau}{\tau_{\mathrm{r}}}}{\left[\frac{4}{9 \tau}+\frac{1}{\tau_{\mathrm{p}}}+\frac{1}{\tau_{\mathrm{r}}}\right]^{2}+\left[\omega-\frac{2}{3} \omega_{\mathrm{m}}\right]^{2}}, \\ F^{\prime}\left(\omega_{1}\right)=\left(\frac{4 \omega_{1}}{3}\right)^{2} \frac{\frac{7}{9}+\frac{\tau}{\tau_{\mathrm{p}}^{\prime}}+\frac{\tau}{\tau_{\mathrm{r}}}}{\left[\frac{7}{9 \tau}+\frac{1}{\tau_{\mathrm{p}}^{\prime}}+\frac{1}{\tau_{\mathrm{r}}}\right]^{2}+\left[\omega-\frac{4}{3} \omega_{\dot{\mathrm{m}}}\right]^{2}} .\end{array}\right.$

Il suffit donc de remplacer dans (2.7) et (2.8) $1 / \tau_{\mathrm{p}}$ et $1 / \tau_{\mathrm{p}}^{\prime}$ par $1 / \tau_{\mathrm{p}}^{*}$ et $1 / \tau_{\mathrm{p}}^{\prime *}$, respectivement pour obtenir les valeurs à l'équilibre de $\left\langle F_{z}\right\rangle_{3 / 2}$ et $\left\langle F_{z}\right\rangle_{1 / 2}$ (il n'est pas possible d'utiliser les expressions simplifiées (2.9) car, si $\omega_{1}$ est grand, $\tau / \tau_{\mathbf{p}}^{*}$ et $\tau / \tau_{\mathbf{p}}^{\prime *}$ ne sont pas très petits devant 1 ).

Il ne reste maintenant plus qu'à reporter ces résultats dans l'équation d'évolution de $\langle\mathbf{I}\rangle_{\mathrm{r}}$. En fait, cette fois encore, le calcul du paragraphe 2 est directement applicable. Cette simplification provient de ce que la seule différence entre les équations d'évolution de $\langle\mathbf{I}\rangle_{\mathrm{f}}$ dans le cas qui nous intéresse ici et dans le cas du paragraphe 2 est due aux termes en $\Omega_{1}$ dans les troisièmes lignes de $(4.3 a)$ et $(4.3 b)$, termes qui traduisent l'action du champ tournant $\mathbf{B}_{1}(t)$ dans le niveau fondamental, et dont l'effet est négligeable. En effet, on a soit $\omega \simeq \frac{2}{3} \omega_{\mathrm{m}}$ (si la résonance a lieu dans le sous-niveau $F=\frac{3}{2}$ ), soit $\omega \simeq \frac{4}{3} \omega_{\mathrm{m}}$ (résonance dans $F=\frac{1}{2}$ ), ce qui entraîne que $\omega$ est très différent de $\omega_{\mathrm{f}}$; de plus $\Omega_{1} \ll \omega_{\mathrm{f}}$ (cette inégalité est identique à (4.7)), ce qui permet de négliger l'action non résonnante de $\mathbf{B}_{1}(t)$ dans le niveau fondamental.

Pour finir, il suffit d'effectuer les substitutions (4.10) dans (2.11), (2.12) et (2.13) pour obtenir l'évolution de $\left\langle I_{z}\right\rangle_{\mathrm{f}}$ et sa valeur limite lorsqu'on effectue la résonance magnétique dans le niveau métastable. Ecrivons de façon explicite les résultats ainsi obtenus.

(i) $\mathrm{Si} \omega \simeq \frac{2}{3} \omega_{\mathrm{m}}$ (résonance sur le sous-niveau $F=\frac{3}{2}$ ), compte tenu de (4.6), la formule $(4.11 b)$ montre que $F^{\prime}\left(\omega_{1}\right) \simeq 0$. Par suite, $\tau_{\mathbf{p}}^{\prime *} \simeq \tau_{\mathbf{p}}^{\prime}$ et la substitution conduit à remplacer l'expression (2.8) de $D$ par

$$
D^{*}=D+a F\left(\omega_{1}\right)
$$

avec

$$
a=\frac{7}{2}+\frac{9}{2}\left(\frac{\tau}{\tau_{\mathrm{r}}}+\frac{\tau}{\tau_{\mathrm{p}}^{\prime}}\right) .
$$

D'après (2.12), on doit alors changer le temps $T_{1}$ de construction de l'orientation nucléaire en $T_{1}^{*}$ tel que :

$$
\begin{aligned}
\frac{1}{T_{1}^{*}}=\frac{1}{T_{\mathrm{r}}}+\frac{1}{T} & \left\{1-\frac{1}{3 D^{*}}\left[3+\frac{11}{2} \frac{\tau}{\tau_{\mathrm{r}}}+\right.\right. \\
+ & \left.\left.\frac{1}{2} \frac{\tau}{\tau_{p}}+\frac{5 \tau}{\tau_{p}^{\prime}}+\frac{1}{2} F\left(\omega_{1}\right)\right]\right\}
\end{aligned}
$$

c'est-à-dire :

$\frac{1}{T_{1}^{*}}=\frac{1}{T_{1}}+\frac{1}{T} F\left(\omega_{1}\right) \frac{a-\frac{1}{6}-a\left(\frac{T}{T_{1}}-\frac{T}{T_{\mathrm{r}}}\right)}{D+a F\left(\omega_{1}\right)}$.

Quant à l'orientation limite $I_{0}$ écrite en (2.13), elle est remplacée par $I_{0}^{*}$ qui vaut, tous calculs faits :

avec :

$$
I_{0}^{*}=I_{0}\left\{1-\left(\frac{2 \omega_{1}}{3}\right)^{2} \frac{\left(K \frac{T_{1}}{T}+\frac{3}{2} C^{\prime}\right)}{\left[\omega-\frac{2}{3} \omega_{\mathrm{m}}\right]^{2}+\left[\frac{4}{9 \tau}+\frac{1}{\tau_{\mathrm{p}}}+\frac{1}{\tau_{\mathrm{r}}}\right]^{2}+K \frac{T_{1}}{T}\left(\frac{2 \omega_{1}}{3}\right)^{2}}\right\}
$$

et

$$
K=\frac{1}{D}\left[a\left(1+\frac{T}{T_{\mathrm{r}}}\right)-\frac{1}{6}\right]\left[\frac{4}{9}+\frac{\tau}{\tau_{\mathrm{p}}}+\frac{\tau}{\tau_{\mathrm{r}}}\right]
$$

$$
C^{\prime}=\frac{\left(\frac{\tau}{\tau_{\mathrm{p}}^{\prime}}\right) \Phi^{\prime}\left[\frac{4}{9}+\frac{\tau}{\tau_{\mathrm{p}}}+\frac{\tau}{\tau_{\mathrm{r}}}\right]}{\left[1+\frac{3}{2} \frac{\tau}{\tau_{\mathrm{r}}}+\frac{3}{2} \frac{\tau}{\tau_{\mathrm{p}}^{\prime}}\right] \frac{\tau}{\tau_{\mathrm{p}}} \Phi+\left[1-\frac{3}{2} \frac{\tau}{\tau_{\mathrm{r}}}-\frac{3}{2} \frac{\tau}{\tau_{\mathrm{p}}}\right] \frac{\tau}{\tau_{\mathrm{p}}^{\prime}} \Phi^{\prime}} .
$$

(ii) $\mathrm{Si} \omega \simeq \frac{4}{3} \omega_{\mathrm{m}}$ (résonance sur le sous-niveau $F=\frac{1}{2}$ ), on a $F\left(\omega_{1}\right) \simeq 0, \tau_{\mathbf{p}}^{*} \simeq \tau_{\mathrm{p}}$, et les quantités $T_{1}$ et $I_{0}$ doivent cette fois être remplacées par $T_{1}^{\prime *}$ et $I_{0}^{\prime *}$ telles que

$$
\begin{gathered}
\frac{1}{T_{1}^{\prime *}}=\frac{1}{T_{1}}+\frac{1}{T} F^{\prime}\left(\omega_{1}\right) \frac{a^{\prime}-\frac{5}{3}-a^{\prime}\left(\frac{T}{T_{1}}-\frac{T}{T_{\mathrm{r}}}\right)}{D+a^{\prime} F^{\prime}\left(\omega_{1}\right)} \\
I_{0}^{\prime *}=I_{0}\left\{1-\left(\frac{4 \omega_{1}}{3}\right)^{2} \frac{K^{\prime} \frac{T_{1}}{T}-\frac{3}{2} C}{\left[\omega-\frac{4}{3} \omega_{\mathrm{m}}\right]^{2}+\left[\frac{7}{9 \tau}+\frac{1}{\tau_{\mathrm{p}}^{\prime}}+\frac{1}{\tau_{\mathrm{r}}}\right]^{2}+K^{\prime} \frac{T_{1}}{T}\left(\frac{4 \omega_{1}}{3}\right)^{2}}\right\}
\end{gathered}
$$


avec :

$$
\begin{gathered}
a^{\prime}=2+\frac{9 \tau}{2 \tau_{\mathrm{r}}}+\frac{9 \tau}{2 \tau_{\mathrm{p}}} \\
K^{\prime}=\frac{1}{D}\left[a^{\prime}\left(1+\frac{T}{T_{\mathrm{r}}}\right)-\frac{5}{3}\right]\left[\frac{7}{9}+\frac{\tau}{\tau_{\mathrm{r}}}+\frac{\tau}{\tau_{\mathrm{p}}^{\prime}}\right] \\
\frac{\frac{\tau}{\tau_{\mathrm{p}}} \Phi\left(\frac{7}{9}+\frac{\tau}{\tau_{\mathrm{p}}^{\prime}}+\frac{\tau}{\tau_{\mathrm{r}}}\right)}{\left[1+\frac{3}{2}\left(\frac{\tau}{\tau_{p}}+\frac{\tau}{\tau_{\mathrm{p}}^{\prime}}\right)\right] \frac{\tau}{\tau_{\mathrm{p}}} \Phi+\left[1-\frac{3}{2}\left(\frac{\tau}{\tau_{\mathrm{r}}}+\frac{\tau}{\tau_{\mathrm{p}}}\right) \frac{\tau}{\tau_{\mathrm{p}}^{\prime}} \Phi^{\prime}\right]} .
\end{gathered}
$$

ß) Discussion des résultats. - Lorsqu'on effectue la résonance magnétique dans l'un des sous-niveaux hyperfins $F=\frac{3}{2}$ ou $F=\frac{1}{2}$, on observe donc une variation de la constante de temps de construction de l'orientation nucléaire dans le fondamental, ainsi que de l'orientation limite obtenue. Compte tenu du fait que, dans la plupart des expériences, on a $\tau / \tau_{\mathrm{r}}, \tau / \tau_{\mathrm{p}}, \tau / \tau_{\mathrm{p}}^{\prime}, T / T_{1}, T / T_{\mathrm{r}} \ll 1$, les formules (4.15) et $(4.19)$ donnent :

$$
\begin{aligned}
& \frac{1}{T_{1}^{*} .} \simeq \frac{1}{T_{1}}+\frac{1}{T}\left(\frac{2 \omega_{1}}{3}\right)^{2} \times \\
& \times \frac{\frac{40}{27}}{\left(\omega-\frac{2}{3} \omega_{\mathrm{m}}\right)^{2}+\left(\frac{4}{9 \tau}\right)^{2}+\frac{14}{9}\left(\frac{2 \omega_{1}}{3}\right)}
\end{aligned}
$$

et :

$$
\begin{aligned}
& \frac{1}{T_{1}^{\prime *}}=\frac{1}{T_{1}}+\frac{1}{T}\left(\frac{4 \omega_{1}}{3}\right)^{2} \times \\
& \times \frac{\frac{7}{27}}{\left[\omega-\frac{4}{3} \omega_{\mathrm{m}}\right]^{2}+\left[\frac{7}{9 \tau}\right]^{2}+\frac{14}{9}\left(\frac{4 \omega_{1}}{3}\right)^{2}} .
\end{aligned}
$$

Ces égalités montrent que le temps de construction de l'orientation nucléaire subit une variation résonnante lorsque $\omega \simeq \frac{2}{3} \omega_{\mathrm{m}}$ ou $\omega \simeq \frac{4}{3} \omega_{\mathrm{m}}$; l'effet de la radiofréquence est de raccourcir ce temps, d'une manière d'autant plus efficace que $\omega$ est plus proche de $\frac{2}{3} \omega_{\mathrm{m}}$ ou de $\frac{4}{3} \omega_{\mathrm{m}}$. Lorsque $\omega_{1}=0$ on trouve $T_{1}^{*}=T_{1}^{\prime *}=T_{1}$, ce qui était prévisible; lorsque $\omega_{1} \tau \gg 1$ (résonances saturées), on trouve :

$$
\begin{aligned}
& {\left[\frac{1}{T_{1}^{*}}\right]_{\omega_{1}=\infty}=\frac{1}{T_{\mathrm{r}}}+\frac{20}{21} \frac{1}{T},} \\
& {\left[\frac{1}{T_{1}^{\prime *}}\right]_{\omega_{1}=\infty}=\frac{1}{T_{\mathrm{r}}}+\frac{1}{6} \frac{1}{T} .}
\end{aligned}
$$

La cinétique du pompage optique est donc profondément affectée par un champ de radiofréquence saturant la résonance du sous-niveau $F=\frac{3}{2}$ : au lieu d'un temps $T_{1}$ de l'ordre de 10 ou 100 secondes, on obtient un temps $T_{1}^{*} \simeq 21 T / 20$ de l'ordre de $\frac{1}{10}$ à 1 seconde (ceci se comprend bien puisque, le niveau $F=\frac{3}{2}$ étant maintenu désorienté, l'échange devient un processus de relaxation pour l'orientation nucléaire du niveau fondamental). En ce qui concerne la résonance sur le sous-niveau $F=\frac{1}{2}$, l'effet d'un champ de radiofréquence est à peu près 6 fois moins prononcé, mais toujours sensible, comme le montre la formule $(4.26 b)$; ce résultat est à rapprocher du fait que l'orientation à l'équilibre du sous-niveau $F=\frac{1}{2}$ est beaucoup plus faible que celle du sous-niveau $F=\frac{3}{2}$.

Les égalités (4.16) et (4.20) donnent les variations résonnantes de l'orientation nucléaire limite sous l'effet du champ de radiofréquence lorsque $\omega \simeq \frac{2}{3} \omega_{\mathrm{m}}$ ou $\omega \simeq \frac{4}{3} \omega_{\mathrm{m}}$. Dans le cas fréquent en pratique où les inégalités $\tau / \tau_{\mathrm{r}}, \tau / \tau_{\mathrm{p}}, \tau / \tau_{\mathrm{p}}^{\prime}, T / T_{\mathrm{r}}$ et $T / T_{1} \ll 1$ sont satisfaites, les formules (4.16) et (4.20) se simplifient $\left(K=40 / 27, K^{\prime}=7 / 27\right):$ on obtient deux courbes de Lorentz, centrées en $\omega=\frac{2}{3} \omega_{\mathrm{m}}$ et $\omega=\frac{4}{3} \omega_{\mathrm{m}}$, de demilargeurs :

$$
\left\{\begin{array}{l}
(\Delta \omega)_{3 / 2} \simeq \sqrt{\left(\frac{4}{9 \tau}\right)^{2}+\frac{40}{27} \frac{T_{1}}{T}\left(\frac{2 \omega_{1}}{3}\right)^{2}} \\
(\Delta \omega)_{1 / 2} \simeq \sqrt{\left(\frac{7}{9 \tau}\right)^{2}+\frac{7}{27} \frac{T_{1}}{T}\left(\frac{4 \omega_{1}}{3}\right)^{2}}
\end{array}\right.
$$

et de hauteurs (normalisées de façon qu'une hauteur de 1 corresponde à une désorientation totale) :

$$
\begin{aligned}
& (h)_{3 / 2} \simeq\left(\frac{2 \omega_{1}}{3}\right)^{2} \frac{\frac{40}{27} \frac{T_{1}}{T}+\frac{3}{2} C^{\prime}}{\left(\frac{4}{9 \tau}\right)^{2}+\frac{40}{27} \frac{T_{1}}{T}\left(\frac{2 \omega_{1}}{3}\right)^{2}}, \\
& (h)_{1 / 2} \simeq\left(\frac{4 \omega_{1}}{3}\right)^{2} \frac{\frac{7}{27} \frac{T_{1}}{T}-\frac{3}{2} C}{\left(\frac{7}{9 \tau}\right)^{2}+\frac{7}{27} \frac{T_{1}}{T}\left(\frac{4 \omega_{1}}{3}\right)^{2}} .
\end{aligned}
$$

Une fois extrapolées à intensité de radiofréquence nulle, les deux demi-largeurs $(\Delta \omega)_{3 / 2}$ et $(\Delta \omega)_{1 / 2}$ valent $4 / 9 \tau$ et $7 / 9 \tau$; elles sont uniquement déterminées par les taux de destruction de l'orientation transversale dans chacun des sous-niveaux $F=\frac{3}{2}$ et $F=\frac{1}{2}$ qui, comme nous l'avons déjà remarqué, dif- 
fèrent tous deux de $1 / \tau$. Il faut donc prendre garde aux facteurs $\frac{4}{9}$ et $\frac{7}{9}$ lorsqu'on veut déduire une valeur de $\tau$, c'est-à-dire de la section efficace d'échange de métastabilité, de mesures des demi-largeurs des courbes de résonance [5], [7]. Ces dernières subissent évidemment un élargissement de radiofréquence qui est donné par les termes en $\omega_{1}^{2}$ dans (4.27).

Comme $T_{1} \gg T$ on ne fait pas une grande erreur en négligeant, dans les hauteurs données par (4.28), les termes en $C$ et $C^{\prime}$. On constate alors que, pour les faibles valeurs de $\omega_{1}$, la résonance du sous-niveau $F=\frac{3}{2}$ est environ $70 / 16 \simeq 4,4$ fois plus intense que celle du sous-niveau $F=\frac{1}{2}$. Par contre, lorsque $\omega_{1} \rightarrow \infty$, les hauteurs des deux résonances tendent vers 1 , et le spin nucléaire est complètement désorienté dans le niveau fondamental $\left({ }^{10}\right)$. Pour obtenir des courbes de résonance qui soient approximativement à mi-saturation, les formules qui donnent les largeurs et les hauteurs montrent qu'il faut prendre une valeur de $\omega_{1}$ de l'ordre de $(1 / \tau) \sqrt{T / T_{1}} ;$ par contre, pour que $T_{1}^{*}$ ou $T_{1}^{\prime *}$ soient de l'ordre de leurs valeurs minimales (4.26) il faut, à résonance, que $\omega_{1}$ soit de l'ordre de $1 / \tau$, c'est-à-dire nettement plus grand puisque $T_{1} \gg T$. Ceci se comprend bien : pour modifier considérablement l'orientation nucléaire limite, il suffit au champ de radiofréquences de raccourcir de façon appréciable l'un des temps $T_{1}^{*}$ ou $T_{1}^{\prime *}$, mais pas nécessairement jusqu'à une valeur de l'ordre du temps d'échange $T$. Il n'est donc pas indispensable qu'il soit assez intense pour détruire en un temps de l'ordre de $\tau$ l'orientation d'un des sousniveaux hyperfins; il suffit qu'il contribue faiblement à sa désorientation mais toutefois assez pour que, à la longue (au bout d'un temps de l'ordre de $T_{1}$ ), ceci se traduise par une destruction de l'orientation du niveau fondamental.

Nous voyons donc que le couplage entre les orientations longitudinales $\left\langle F_{z}\right\rangle_{3 / 2},\left\langle F_{z}\right\rangle_{1 / 2}$ et $\left\langle I_{z}\right\rangle_{\mathrm{f}}$ intervient dans les formes de courbe de résonance d'une façon non évidente a priori. On peut remarquer que les relations $(4.27 a)$ et $(4.28 a)$ sont celles que donneraient les équations de Bloch, pour un niveau

$\left({ }^{10}\right)$ Si nous avions gardé, dans les hauteurs $(h)_{3 / 2}$ et $(h)_{1 / 2}$, les termes en $C$ et $C^{\prime}$, nous aurions trouvé que cette désorientation n'est pas tout à fait totale. Physiquement, ceci s'explique par le fait que, même si la radiofréquence désoriente totalement l'un des sous-niveaux hyperfins, de l'orientation continue à être transférée au niveau fondamental par l'intermédiaire de l'autre sous-niveau hyperfin. isolé de temps de relaxation transversal $\tau_{2}=\frac{9 \tau}{4}$ et longitudinal $\tau_{1}=\frac{10}{3} \frac{T_{1}}{T} \tau$ (pour la résonance dans le niveau $F=\frac{1}{2}$, on aurait $\tau_{2}^{\prime}=\frac{9 \tau}{7}$ et $\left.\tau_{1}^{\prime}=\frac{1}{3} \frac{T_{1}}{T} \tau\right)$; le temps de relaxation longitudinal de ce niveau fictif dépendrait alors, non seulement des propriétés $\mathrm{du}$ niveau métastable, mais également de celles du niveau fondamental (par l'intermédiaire de $T$ et de $T_{1}$ qui fait intervenir $T_{\mathrm{r}}$ ).

Rappelons que toute cette discussion concerne l'observation des résonances pour la mesure du taux de polarisation des raies émises par la décharge, c'est-à-dire par l'intermédiaire de $\left\langle I_{z}\right\rangle_{\mathrm{f}}$. Si l'on observait ces résonances en mesurant l'absorption de la raie $\lambda=10830 \AA$, il faudrait calculer $\left\langle F_{z}\right\rangle_{3 / 2}$ et $\left\langle F_{z}\right\rangle_{1 / 2}$, c'est-à-dire simplement remplacer $\tau_{\mathrm{p}}$ et $\tau_{\mathbf{p}}^{\prime} \operatorname{par} \tau_{\mathbf{p}}^{*}$ et $\tau_{\mathbf{p}}^{\prime *}$ dans (2.7). Les résultats concernant les largeurs de résonances seraient alors les mêmes, mais ceux concernant leurs hauteurs seraient modifiés.

4.3.2 Effets liés à la circulation de cohérence. Lorsque $\omega_{\mathrm{m}} \tau \simeq 1$, il peut y avoir circulation de cohérence entre les deux sous-niveaux $F=\frac{3}{2}$ et $F=\frac{1}{2}$, et les éq. (4.9) ne sont plus valables. Nous n'allons pas donner ici un calcul des formes de courbe de résonance magnétique dans le cas général, qui conduirait à des calculs trop compliqués. Nous nous contenterons d'étudier le cas où $\omega_{\mathrm{m}} \tau$ est toujours assez élevé pour qu'on puisse se limiter aux corrections d'ordre le plus bas (en $1 / \omega_{\mathrm{m}} \tau$ ) au calcul du paragraphe 4.3.1 précédent, ce qui nous permettra de discuter qualitativement le type d'effets que peut entraîner la circulation de cohérence. En général, les expériences sont effectivement faites dans ce cas : le champ $B_{0}$ est assez élevé pour séparer les résonances des sous-niveaux $F=\frac{3}{2}$ et $F=\frac{1}{2}$, ce qui permet de mesurer facilement leurs positions. L'intérêt pratique d'un calcul au premier ordre en $1 / \omega_{m} \tau$ est donc de fournir la valeur des corrections (modification des positions des résonances) qui dans une expérience, peuvent provenir de la circulation de cohérence; ceci est évidemment essentiel dans toute mesure de précision des facteurs de Landé des différents niveaux. Dans un but de simplification, nous négligerons dans tout ce paragraphe les termes en $\tau / \tau_{\mathbf{r}}, \tau / \tau_{\mathbf{p}}, \tau / \tau_{\mathbf{p}}^{\prime}, T / T_{\mathrm{r}}$ et $T / T_{1}$.

Les équations couplées d'évolution des orientations transversales sont alors :

$$
\left\{\begin{array}{l}
\frac{\mathrm{d}}{\mathrm{d} t} j_{3 / 2}^{+}=-\left[\frac{4}{9 \tau}+i\left(\omega-\frac{2}{3} \omega_{\mathrm{m}}\right)\right] j_{3 / 2}^{+}+\frac{10}{9 \tau} j_{1 / 2}^{+}+\frac{10}{9 \tau} j_{\mathrm{f}}^{+}+\frac{2 \omega_{1}}{3}\left\langle F_{z}\right\rangle_{3 / 2} \\
\frac{\mathrm{d}}{\mathrm{d} t} j_{1 / 2}^{+}=\frac{1}{9 \tau} j_{3 / 2}^{+}-\left[\frac{7}{9 \tau}+i\left(\omega-\frac{4}{3} \omega_{\mathrm{m}}\right)\right] j_{1 / 2}^{+}-\frac{1}{9 \tau} j_{\mathrm{f}}^{+}+\frac{4 \omega_{1}}{3}\left\langle F_{z}\right\rangle_{1 / 2} \\
\frac{\mathrm{d}}{\mathrm{d} t} j_{\mathrm{f}}^{+}=\frac{1}{3 T} j_{3 / 2}^{+}-\frac{1}{3 T} j_{1 / 2}^{+}-\left[\frac{1}{T}+i\left(\omega-\omega_{\mathrm{f}}\right)\right] j_{\mathrm{f}}^{+}+\Omega_{1}\left\langle I_{z}\right\rangle_{\mathrm{f}} .
\end{array}\right.
$$


Supposons que

$$
\left\{\begin{array}{l}
\omega \simeq \frac{2}{3} \omega_{\mathrm{m}} \\
\omega_{\mathrm{m}} \tau \gg 1
\end{array}\right.
$$

(on effectue la résonance dans le sous-niveau $F=\frac{3}{2}$; les raisonnements pour le sous-niveau $F=\frac{1}{2}$ seraient analogues), et cherchons les solutions stationnaires de (4.29) aux ordres les plus bas en

$$
\frac{1}{\omega_{m} \tau}, \frac{\omega-\frac{2}{3} \omega_{m}}{\omega_{m}}, \frac{\omega_{1}}{\omega_{m}}
$$

(nous avons vu dans le paragraphe 4.3.1 précédent que, pour observer la résonance, il faut un champ $B_{1}$ tel que $\left.\omega_{1} \lesssim 1 / \tau\right)$. A l'ordre zéro en $1 / \omega_{\mathrm{m}} \tau$, nous avons [cf. (4.29)] :

$$
\left\{\begin{aligned}
j_{3 / 2}^{+} & \simeq \frac{2 \omega_{1} / 3}{\frac{4}{9 \tau}+i\left(\omega-\omega_{\mathrm{m}}\right)}\left\langle F_{z}\right\rangle_{3 / 2} \\
j_{1 / 2}^{+} & \simeq 0 \\
j_{\mathrm{f}}^{+} & \simeq 0
\end{aligned}\right.
$$

(seule, l'orientation transversale du sous-niveau $F=\frac{3}{2}$ où l'on effectue la résonance n'est pas nulle). A l'ordre un en $1 / \omega_{\mathrm{m}} \tau, \omega_{1} / \omega_{\mathrm{m}}$, les corrections à (4.31) s'obtiennent aisément pour $j_{1 / 2}^{+}$et $j_{\mathrm{f}}^{+}$en reportant $(4.31)$ dans (4.29); il vient, compte tenu de (4.30) et du fait que $\omega_{\mathrm{f}} \ll \omega_{\mathrm{m}}$

$$
\begin{aligned}
j_{1 / 2}^{+} & \simeq \frac{\frac{1}{9 \tau} j_{3 / 2}^{+}+\frac{4 \omega_{1}}{3}\left\langle F_{z}\right\rangle_{1 / 2}}{i\left(\omega-\frac{4}{3} \omega_{\mathrm{m}}\right)} \\
& \simeq i \frac{j_{3 / 2}^{+}}{6 \omega_{\mathrm{m}} \tau}+i \frac{2 \omega_{1}}{\omega_{\mathrm{m}}}\left\langle F_{z}\right\rangle_{1 / 2}
\end{aligned}
$$

et :

$$
\begin{aligned}
j_{\mathrm{f}}^{+} & \simeq \frac{\frac{1}{3 T} j_{3 / 2}^{+}+\Omega_{1}\left\langle I_{z}\right\rangle_{\mathrm{f}}}{i\left(\omega-\omega_{\mathrm{f}}\right)} \\
& \simeq-i \frac{j_{3 / 2}^{+}}{2 \omega_{\mathrm{m}} T}-\frac{3}{2} i \frac{\Omega_{1}}{\omega_{\mathrm{m}}}\left\langle I_{z}\right\rangle_{\mathrm{f}} .
\end{aligned}
$$

Comme $T \gg \tau, \Omega_{1} \ll \omega_{1}$, la correction du premier ordre de $j_{\mathrm{f}}^{+}$est négligeable $\left(j_{\mathrm{f}}^{+} \simeq 0\right)$ et nous ne retiendrons que celle de $j_{1 / 2}^{+}$écrite en (4.32). En reportant cette égalité dans la première ligne de (4.29), on obtient la valeur de $j_{3 / 2}^{+}$au premier ordre en $1 / \omega_{\mathrm{m}} \tau, \omega_{1} / \omega_{\mathrm{m}}$ :

$j_{3 / 2}^{+} \simeq \frac{\frac{2 \omega_{1}}{3}\left\langle F_{z}\right\rangle_{3 / 2}+i \frac{20}{9 \tau} \frac{\omega_{1}}{\omega_{\mathrm{m}}}\left\langle F_{z}\right\rangle_{1 / 2}}{\frac{4}{9 \tau}+i\left(\omega-\frac{2}{3} \omega_{\mathrm{m}}-\frac{5}{27} \frac{1}{\omega_{\mathrm{m}} \tau^{2}}\right)}$

et, par suite :

$$
\begin{aligned}
\operatorname{Re} j_{3 / 2}^{+}=\frac{3}{2 \omega_{1} \tau} & \tilde{F}\left(\omega_{1}\right)\left\langle F_{z}\right\rangle_{3 / 2}+ \\
& +\frac{10}{3 \omega_{\mathrm{m}} \tau} \tilde{G}\left(\omega_{1}\right)\left\langle F_{z}\right\rangle_{1 / 2} .
\end{aligned}
$$

Dans cette expression, $\widetilde{F}\left(\omega_{1}\right)$ est obtenue en changeant, dans l'expression (4.11a) de $F\left(\omega_{1}\right), \frac{2}{3} \omega_{\mathrm{m}}$ en $\frac{2}{3} \omega_{\mathrm{m}}+\delta$, avec :

$$
\delta=\frac{5}{27} \frac{1}{\omega_{\mathrm{m}} \tau^{2}}
$$

et $\widetilde{G}\left(\omega_{1}\right)$ est défini par :

$$
\tilde{G}\left(\omega_{1}\right)=\frac{\frac{2 \omega_{1}}{3}\left(\omega-\frac{2}{3} \omega_{m}-\delta\right)}{\left(\frac{4}{9 \tau}\right)^{2}+\left[\omega-\frac{2}{3} \omega_{m}-\delta\right]^{2}} .
$$

Avec ces notations, en reportant (4.34) dans (4.32), et en négligeant les termes en $\left(1 / \omega_{\mathrm{m}} \tau\right)^{2}$, on obtient :

$$
\operatorname{Re} j_{1 / 2}^{+}=\frac{1}{6 \omega_{\mathrm{m}} \tau} \tilde{G}\left(\omega_{1}\right)\left\langle F_{z}\right\rangle_{3 / 2}
$$

Il reste à reporter (4.35) et (4.38) dans les équations d'évolution des orientations longitudinales pour obtenir leurs valeurs stationnaires. Il vient ainsi :

$$
\left\{\begin{aligned}
- & {\left[\frac{4}{9 \tau}+\frac{\tilde{F}(\omega)}{\tau}\right]\left\langle F_{z}\right\rangle_{3 / 2}+10\left[\frac{1}{9 \tau}-\frac{2 \omega_{1}}{9 \omega_{\mathrm{m}} \tau} \tilde{G}\left(\omega_{1}\right)\right]\left\langle F_{z}\right\rangle_{1 / 2}+\frac{10}{9 \tau}\left\langle I_{z}\right\rangle_{\mathrm{f}}+\frac{\Phi}{\tau_{\mathrm{p}}}=0 } \\
& {\left[\frac{1}{9 \tau}-\frac{2 \omega_{1}}{9 \omega_{\mathrm{m}} \tau} \tilde{G}\left(\omega_{1}\right)\right]\left\langle F_{z}\right\rangle_{3 / 2}-\frac{7}{9 \tau}\left\langle F_{z}\right\rangle_{1 / 2}-\frac{1}{9 \tau}\left\langle I_{z}\right\rangle_{\mathrm{f}}+\frac{\Phi^{\prime}}{\tau_{\mathrm{p}}^{\prime}}=0 . }
\end{aligned}\right.
$$

La solution de ce système s'écrit :

$$
\begin{aligned}
& \left\langle F_{z}\right\rangle_{3 / 2}=\frac{1}{\widetilde{D}}\left\{\left[\frac{10}{3}+\frac{10}{9} \frac{\omega_{1}}{\omega_{\mathrm{m}}} \tilde{G}\left(\omega_{1}\right)\right]\left\langle I_{z}\right\rangle_{\mathrm{f}}+\frac{7}{2} \frac{\tau}{\tau_{\mathrm{p}}} \Phi+\left[5-10 \frac{\omega_{1}}{\omega_{\mathrm{m}}} \tilde{G}\left(\omega_{1}\right)\right] \frac{\tau \Phi^{\prime}}{\tau_{\mathrm{p}}^{\prime}}\right\} \\
& \left\langle F_{z}\right\rangle_{1 / 2}=\frac{1}{\tilde{D}}\left\{\left[\frac{1}{3}-\frac{1}{2} \tilde{F}\left(\omega_{1}\right)-\frac{10}{9} \frac{\omega_{1}}{\omega_{\mathrm{m}}} \tilde{G}\left(\omega_{1}\right)\right]\left\langle I_{z}\right\rangle_{\mathrm{f}}+\left[\frac{1}{2}-\frac{\omega_{1}}{\omega_{\mathrm{m}}} \tilde{G}\left(\omega_{1}\right)\right] \frac{\tau \Phi}{\tau_{\mathrm{p}}}+\left[2+\frac{9}{2} \tilde{F}\left(\omega_{1}\right)\right] \frac{\tau \Phi^{\prime}}{\tau_{\mathrm{p}}^{\prime}}\right\}
\end{aligned}
$$


avec :

$$
\tilde{D}=1+\frac{7}{2} \tilde{F}\left(\omega_{1}\right)+\frac{20}{9} \frac{\omega_{1}}{\omega_{\mathrm{m}}} \tilde{G}\left(\omega_{1}\right) .
$$

Ces égalités, reportées dans celle qui donne l'évolution de $\left\langle I_{z}\right\rangle_{\mathrm{f}}$, conduisent à :

avec :

$$
\frac{\mathrm{d}}{\mathrm{d} t}\left\langle I_{z}\right\rangle_{\mathrm{f}}=-\frac{1}{\tilde{T}_{1}}\left\langle I_{z}\right\rangle_{\mathrm{f}}+\frac{\tilde{I}_{0}}{\tilde{T}_{1}}
$$

$\frac{1}{\tilde{T}_{1}}=\frac{1}{T_{\mathrm{r}}}+\frac{1}{T}\left\{1-\frac{1}{\widetilde{D}}\left[1+\frac{\tilde{F}\left(\omega_{1}\right)}{6}+\frac{20}{27} \frac{\omega_{1}}{\omega_{\mathrm{m}}} \tilde{G}\left(\omega_{1}\right)\right]\right\}=\frac{1}{T_{\mathrm{r}}}+\frac{1}{T} \frac{\frac{10}{3} \tilde{F}\left(\omega_{1}\right)+\frac{40}{27} \frac{\omega_{1}}{\omega_{\mathrm{m}}} \tilde{G}\left(\omega_{1}\right)}{1+\frac{7}{2} \tilde{F}\left(\omega_{1}\right)+\frac{20}{9} \frac{\omega_{1}}{\omega_{\mathrm{m}}} \tilde{G}\left(\omega_{1}\right)}$

et

$$
\tilde{I}_{0}=\frac{\tilde{T}_{1}}{\tilde{D} T}\left\{\left[1+\frac{1}{3} \frac{\omega_{1}}{\omega_{\mathrm{m}}} \tilde{G}\left(\omega_{1}\right)\right] \frac{\tau \Phi}{\tau_{\mathrm{p}}}+\left[1-\frac{3}{2} \tilde{F}\left(\omega_{1}\right)-\frac{10}{3} \frac{\omega_{1}}{\omega_{\mathrm{m}}} \tilde{G}\left(\omega_{1}\right)\right] \frac{\tau \Phi^{\prime}}{\tau_{\mathrm{p}}^{\prime}}\right\}
$$

Tous calculs faits, il vient :

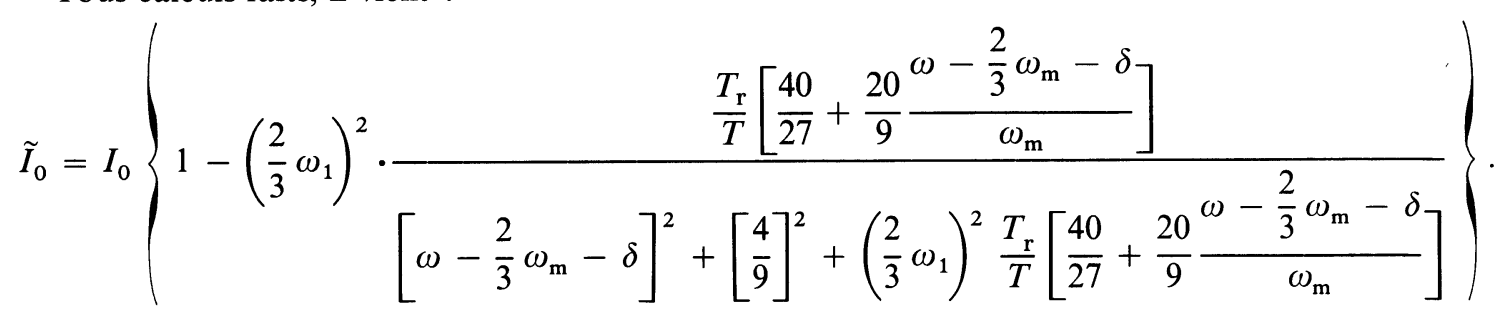

Cette formule est à comparer à l'expression (4.16) obtenue au paragraphe 4.3.1 en négligeant les effets de circulation de cohérence entre les sous-niveaux $F=\frac{1}{2}$ et $F=\frac{3}{2}$ [dans (4.16) on fera les mêmes approximations $\tau / \tau_{\mathrm{r}}, \tau / \tau_{\mathrm{p}}, \tau / \tau_{\mathrm{p}}^{\prime}, T / T_{\mathrm{r}} \ll 1$, que dans tout ce paragraphe 4.3 .2 ; on a alors $K=\frac{40}{27}$ et $\left.C^{\prime}=0\right]$.

Les termes nouveaux qui apparaissent dans (4.45) entraînent un déplacement de la résonance et une modification de sa forme. En effet, en mettant sous forme canonique le trinôme du second degré en $\omega$ au dénominateur de (4.45), on trouve que la condition de résonance est réalisée pour :

$$
\omega=\frac{2}{3} \omega_{\mathrm{m}}+\delta+\delta_{1}
$$

où $\delta$ est donné par (4.36); $\delta_{1}$ est défini par :

$$
\delta_{1}=-\left(\frac{2 \omega_{1}}{3}\right)^{2} \times \frac{10}{9} \times \frac{T_{\mathrm{r}}}{T} \times \frac{1}{\omega_{\mathrm{m}}} .
$$

Si l'on se reporte à la formule (3.4), on constate que le terme $\delta$ représente simplement, au premier ordre en $1 / \omega_{\mathrm{m}} \tau$, le déplacement de la fréquence propre du métastable $F=\frac{3}{2}$ sous l'effet de la circulation de cohérence avec le niveau $F=\frac{1}{2}$ induite par l'échange de métastabilité en l'absence de champ de radiofréquence. Si l'on extrapole la position de la résonance à $\omega_{1}=0$, le seul déplacement de cette position est $\delta$.

Par contre, dès que $\omega_{1} \neq 0$, il faut tenir compte du second terme en $\delta_{1}$ qui apparaît dans l'éq. (4.46); $\delta_{1}$ est de signe opposé à $\delta$ et quadratique en $\omega_{1}$. L'origine physique de ce déplacement de fréquence $\delta_{1}$ est l'action non résonnante du champ $\mathbf{B}_{1}(t)$ dans le sous-niveau hyperfin $F=\frac{1}{2}$ lorsqu'on effectue la résonance dans le sous-niveau $F=\frac{3}{2}\left({ }^{11}\right)$.

Il est intéressant de comparer ces déplacements de fréquence avec la largeur $(\Delta \omega)_{3 / 2}$ de la courbe de résonance, donnée par la formule $(4.27 a)$. A mi-saturation de la résonance $\left(\omega_{1} \sim(1 / \tau) \sqrt{T_{\mathrm{r}} / T}\right.$, cf. $\S 4.3 .1 \beta)$, on a :

$$
\frac{\delta}{(\Delta \omega)_{3 / 2}} \simeq-\frac{\delta_{1}}{(\Delta \omega)_{3 / 2}} \simeq \frac{1}{\omega_{\mathrm{m}} \tau}
$$

Les deux déplacements sont donc du même ordre de grandeur mais de signe opposé.

(11) Il ne faut pas confondre $\delta_{1}$ avec un déplacement de BlochSiegert de la résonance, qui n'existe pas lorsque $\mathbf{B}_{1}(t)$ est un champ tournant comme nous l'avons supposé ici. Avec un champ linéaire oscillant, le déplacement Bloch-Siegert serait de l'ordre de $\omega_{1}^{2} / \omega_{\mathrm{m}}$, c'est-à-dire nettement inférieur à $\delta_{1}$ puisque $T_{1} \gg T$ (on a couramment $T_{1} \gg 100 T$, cf. Tableau I). 
Enfin, on constate sur (4.45) que la courbe de résonance n'est plus une lorentzienne pure; c'est la somme d'une courbe d'absorption et d'une courbe de dispersion, centrées à la même valeur de $\omega$; aux faibles valeurs de $\omega_{1}$, le rapport des amplitudes de la dispersion et de l'absorption est de $2 / 3 \omega_{\mathrm{m}} \tau$.

Un calcul analogue peut être fait pour la résonance dans le sous-niveau $F=\frac{1}{2}$. On trouve

$$
\tilde{I}_{0}^{\prime}=I_{0}\left\{1-\left(\frac{4}{3} \omega_{1}\right)^{2} \frac{\frac{T_{\mathrm{r}}}{T}\left[\frac{7}{27}-\frac{5}{9} \frac{\omega-\frac{4}{3} \omega_{\mathrm{m}}-\delta^{\prime}}{\omega_{\mathrm{m}}}\right]}{\left(\omega-\frac{4}{3} \omega_{\mathrm{m}}-\delta^{\prime}\right)^{2}+\left(\frac{7}{9 \tau}\right)^{2}+\left(\frac{4}{3} \omega_{1}\right)^{2} \frac{T_{\mathrm{r}}}{T}\left[\frac{7}{27}-\frac{5}{9} \frac{\omega-\frac{4}{3} \omega_{\mathrm{m}}-\delta^{\prime}}{\omega_{\mathrm{m}}}\right]}\right\} .
$$

Le déplacement de la résonance est alors $\delta^{\prime}+\delta_{1}^{\prime}$ avec

$$
\delta^{\prime}=-\frac{5}{27} \times \frac{1}{\omega_{\mathrm{m}} \tau^{2}}
$$

et

$$
\delta_{1}^{\prime}=+\left(\frac{4}{3} \omega_{1}\right)^{2} \times \frac{5}{18} \frac{T_{\mathrm{r}}}{T} \times \frac{1}{\omega_{\mathrm{m}}} .
$$

5. Vérifications expérimentales. - Plusieurs des effets prévus par la théorie développée dans les précédents paragraphes ont fait l'objet de vérifications expérimentales, tant dans notre laboratoire que par d'autres auteurs. Nous n'envisageons pas de les décrire tous ici en détail et nous nous contenterons de présenter un résumé des principaux résultats que nous avons obtenus par des expériences de résonance magnétique dans les niveaux fondamental et métastable de l'hélium; nous pourrons ainsi les comparer aux prévisions théoriques du paragraphe 4. Nous nous limiterons à une description très brève de ces expériences, en renvoyant aussi souvent que possible aux différentes publications où elles sont commentées plus en détail.

Le schéma du montage expérimental a été donné au début du précédent article (voir Fig. 2); le même dispositif a été utilisé pour l'étude des résonances dans les deux sous-niveaux hyperfins du métastable aussi bien que dans le niveau fondamental. Cependant, dans ce dernier cas, la mise en évidence des déplacements de fréquence de la résonance nucléaire implique des valeurs très faibles du champ magnétique $B_{0}$ $\left(B_{0}<0,1 \mathrm{G}\right)$, si bien que cette expérience a été réalisée à l'intérieur d'un blindage magnétique.

\subsection{RÉSONANCES MAGNÉTIQUES DANS LE NIVEAU} MÉTASTABLE. - Ces expériences sont décrites en détail dans la référence [4]; certains résultats ont été publiés dans [5]. Nous les exposons ici brièvement.

La fréquence $\omega / 2 \pi$ du champ oscillant a été choisie égale à $23 \mathrm{MHz}$, de sorte que les résonances des sousniveaux hyperfins $F=\frac{3}{2}$ et $F=\frac{1}{2}$ du métastable $2{ }^{3} \mathrm{~S}_{1}$ soient très bien séparées [ceci correspond à la condition (4.6)]. Nous avons rassemblé sur les figures 5 et 6 les résultats ainsi obtenus avec une cellule contenant 1,45 torr $\mathrm{de}{ }^{3} \mathrm{He}$; en abscisses figure le carré de l'intensité du champ magnétique oscillant $\omega_{1}$ (en unités arbitraires); en ordonnées, sur la figure $5, \Delta B$ représente la largeur (en gauss) mesurée pour chacun des sous-niveaux hyperfins; la figure 6 rend compte de la hauteur $(h)_{3 / 2}$ et $(h)_{1 / 2}$ de ces deux résonances : nous y avons porté la combinaison $y$ de ces deux quantités :

$$
y=\frac{(h)_{1 / 2}}{(h)_{3 / 2}-(h)_{1 / 2}}
$$

dont l'expression théorique est particulièrement simple. Comparons ces résultats expérimentaux aux prévisions du paragraphe 4.3.1.

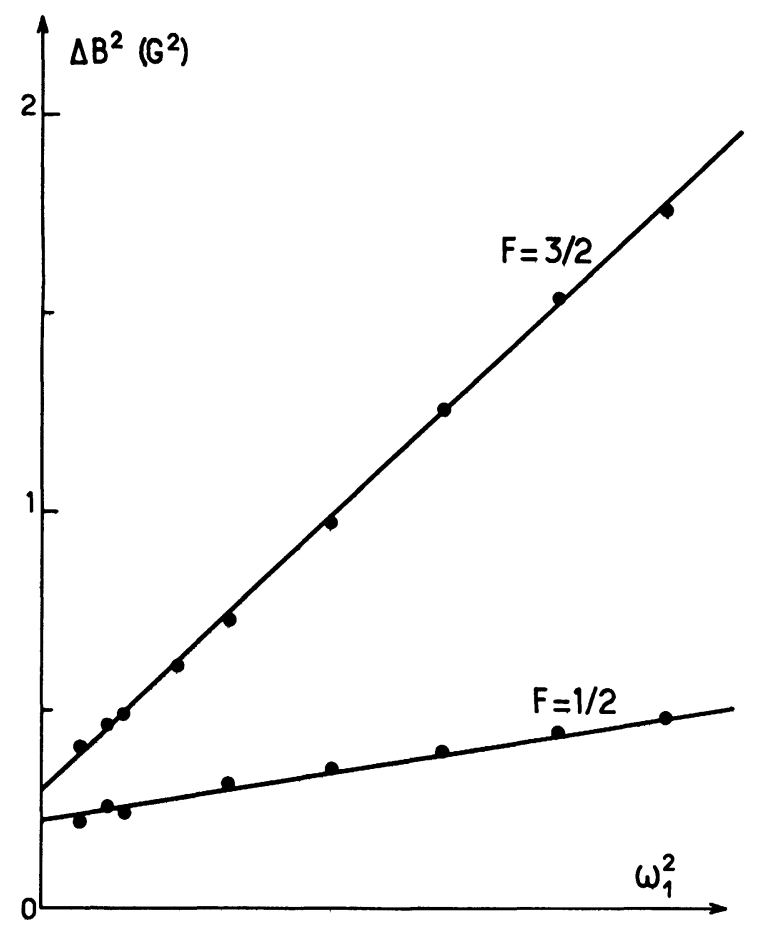

Fig. 5. - Largeur $\Delta B$ des résonances dans les deux sous-niveaux hyperfins $F=\frac{3}{2}$ et $F=\frac{1}{2}$ du niveau métastable $2{ }^{3} \mathrm{~S}_{1}$ de ${ }^{3} \mathrm{He}$. En abscisses est porté le carré de l'intensité $\omega_{1}$ du champ de radiofréquence (pression de ${ }^{3} \mathrm{He}$ dans la cellule : 1,45 torr). 


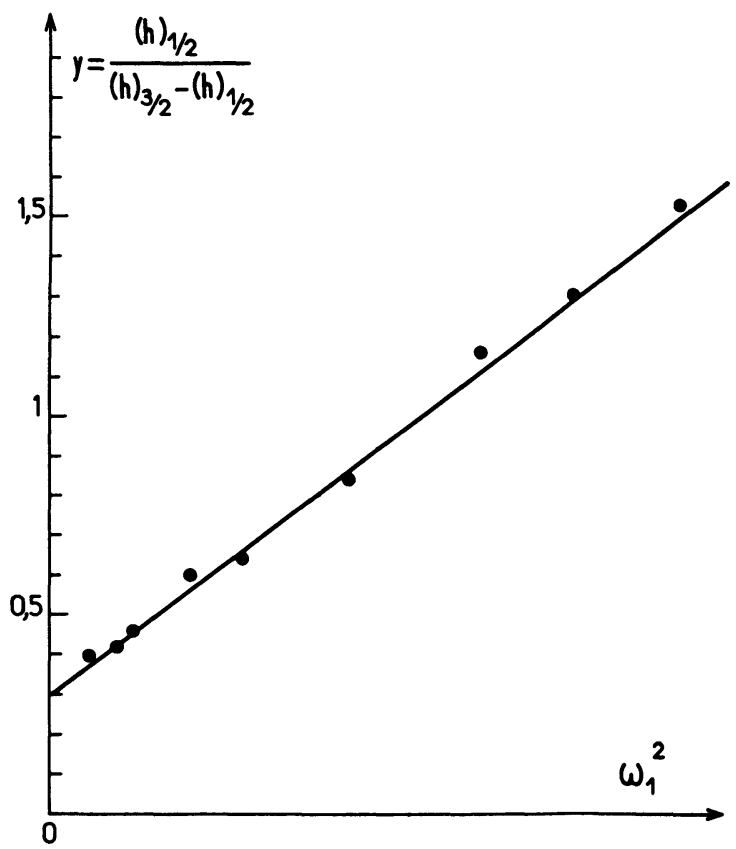

FIG. 6. - $(h)_{1 / 2}$ et $(h)_{3 / 2}$ sont les hauteurs mesurées pour les résonances dans les deux sous-niveaux hyperfins du niveau métastable $2{ }^{3} \mathrm{~S}_{1}$ de ${ }^{3} \mathrm{He}$. En abscisses est porté le carré de l'intensité $\omega_{1}$ du champ de radiofréquence (pression de ${ }^{3} \mathrm{He}$ dans la cellule :

$$
1,45 \text { torr). }
$$

5.1.1 Largeur des résonances. - $\propto$ ) Elargissement en fonction de $\omega_{1}$. - On constate bien sur la figure 5 un accroissement linéaire de $\Delta B^{2}$ avec $\omega_{1}^{2}$, en accord avec les formules $(4.27 a)$ et $(4.27 b)$.

Appelons ${ }^{\circ} \Delta B$ les largeurs $\Delta B$ extrapolées à $\omega_{1}=0$; le rapport des pentes des deux droites de la figure 5 vaut :

$$
\frac{(\Delta B)_{3 / 2}^{2}-\left({ }^{\circ} \Delta B\right)_{3 / 2}^{2}}{(\Delta B)_{1 / 2}^{2}-\left({ }^{\circ} \Delta B\right)_{1 / 2}^{2}}=5,69 \pm 0,4
$$

En unités de pulsation, compte tenu des facteurs de Landé différents pour les 2 niveaux, ce rapport expérimental vaut : $(1,43 \pm 0,1)$. Il est en excellent accord avec sa valeur théorique immédiatement déduite de (4.27) : $\frac{10}{7}=1,428$.

B) Largeurs extrapolées à $\omega_{1}=0$. - Sur la figure 5, on lit les valeurs de ${ }^{\circ} \Delta B$ exprimées en gauss :

$$
\frac{\left({ }^{\circ} \Delta B\right)_{3 / 2}}{\left({ }^{\circ} \Delta B\right)_{1 / 2}}=(1,14 \pm 0,04) .
$$

En unités de pulsation, ce rapport vaut

$$
(0,57 \pm 0,02) \text {. }
$$

Il est à comparer avec sa valeur théorique déduite de (4.27):

$$
\frac{\left({ }^{\circ} \Delta \omega\right)_{3 / 2}}{\left({ }^{\circ} \Delta \omega\right)_{1 / 2}}=\frac{\frac{4}{9} \tau}{\frac{7}{9} \tau}=\frac{4}{7}=0,571 .
$$

On constate que l'accord théorie-expérience est très bon.

Nous avons répété cette étude pour différentes valeurs de la pression $p$ d'hélium dans la cellule. Sur la figure 7 , nous avons porté les largeurs $\left({ }^{\circ} \Delta B\right)_{3 / 2}$ et $\left({ }^{\circ} \Delta B\right)_{1 / 2}$ en fonction de $p$. On constate que pour les deux niveaux $F=\frac{3}{2}$ et $F=\frac{1}{2}$, ces largeurs sont proportionnelles à la pression : ceci tient au fait que l'échange de métastabilité est le processus dominant pour l'évolution du métastable $2{ }^{3} \mathrm{~S}_{1}$. Le rapport des pentes des deux droites est ainsi obtenu avec une très bonne précision $(0,579 \pm 0,012$ en unités de pulsation), en très bon accord avec la valeur théorique. Ce résultat confirme donc entièrement les prévisions des paragraphes 1 et 4 relatives à la conservation partielle de la cohérence dans chacun des niveaux hyperfins lors d'une collision d'échange de métastabilité [facteur $\frac{4}{9}$ et $\frac{7}{9}$ des formules (4.27)]. Rappelons que nous en avons déduit une nouvelle détermination de la valeur de la section efficace de l'échange de métastabilité dans ${ }^{3} \mathrm{He}$ [5] à la température ordinaire :

$$
\sigma=(7,6 \pm 0,4) \times 10^{-16} \mathrm{~cm}^{2}
$$

en net désaccord avec celle admise antérieurement [6].

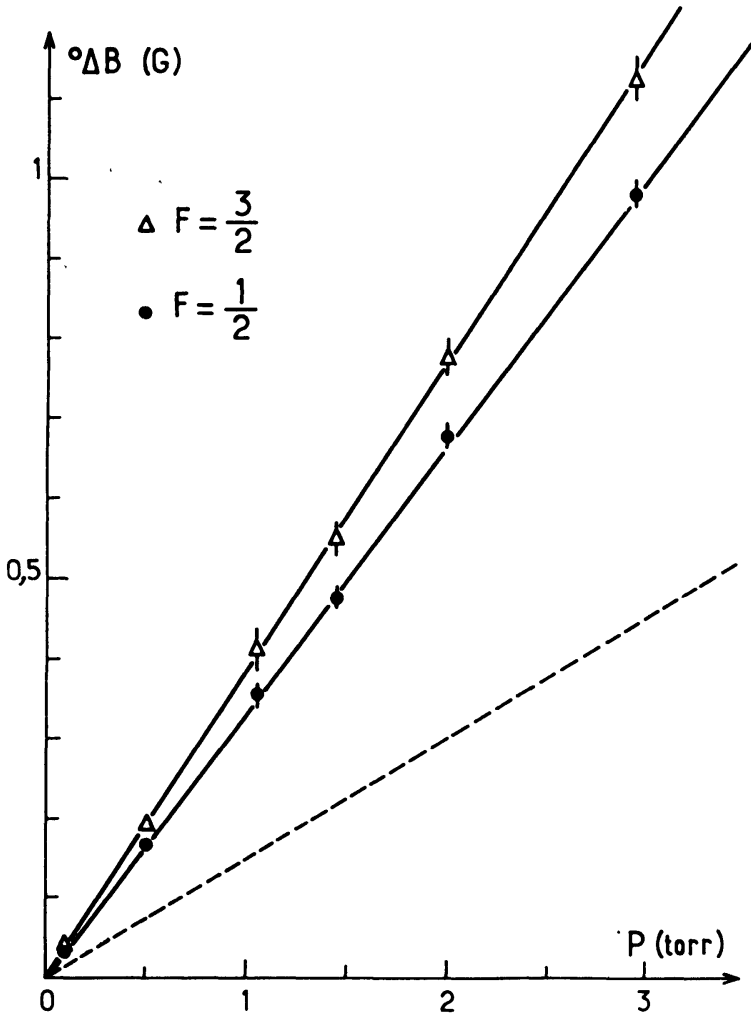

Fig. 7. - Largeurs ${ }^{\circ} \Delta B$ des résonances dans les deux sous-niveaux hyperfins du niveau métastable $2{ }^{3} \mathrm{~S}_{1}$ de ${ }^{3} \mathrm{He}$; ces largeurs, extrapolées à intensité nulle du champ de radiofréquence, sont portées en fonction de la pression $p$ de ${ }^{3} \mathrm{He}$ dans la cellule. Leur rapport vaudrait 2 si les cohérences étaient détruites à chaque collision; dans ce cas les points expérimentaux relatifs au niveau $F=\frac{1}{2}$ seraient sur la droite en pointillés. 
5.1.2 Intensité des résonances. - La valeur théorique de la quantité

$$
y=\frac{(h)_{1 / 2}}{(h)_{3 / 2}-(h)_{1 / 2}}
$$

est déduite des formules (4.28) :

$$
y \simeq \frac{8}{27}+\frac{80}{81} \frac{T_{1}}{T} \tau^{2} \omega_{1}^{2} .
$$

On voit sur la figure 6 que les valeurs expérimentales de $y$ varient bien linéairement avec $\omega_{1}^{2}$. L'ordonnée à l'origine de la droite vaut $(0,295 \pm 0,01)$ : ceci est en bon accord avec la valeur théorique $\frac{8}{27}=0,296$; cette quantité caractérise physiquement le comportement des intensités des 2 résonances aux faibles valeurs de $\omega_{1}$ (loin de la saturation) : celle du niveau $F=\frac{3}{2}$ est beaucoup plus intense que celle du niveau $F=\frac{1}{2}$.

\subsection{RÉSONANCE MAGNÉTIQUE DU NIVEAU FONDA-} MENTAL DE ${ }^{3} \mathrm{He}$ [2], [3]. - Le principal but de cette expérience a été la vérification quantitative détaillée des effets liés à la circulation de cohérence avec le niveau métastable sous l'effet des collisions d'échange. Nous avons vu au paragraphe 4.2 (formule (4.5)) que ceci produit un déplacement $\delta \omega_{\mathrm{f}}$ de la résonance nucléaire de l'état fondamental, ainsi qu'un affinement de la largeur $1 / T_{2}$ de cette résonance extrapolée à $\omega_{1}=0$. Les expressions théoriques de $\delta \omega_{\mathrm{f}}$ et $1 / T_{2}$ en fonction du champ magnétique ont été données en (3.8) et (3.9). La figure 8 montre un exemple de la mise en évidence expérimentale de ces deux effets : les différentes courbes de résonance nucléaire de ${ }^{3} \mathrm{He}$ enregistrées correspondent à des intensités de décharge croissantes (repérées sur la figure par la valeur du paramètre $i$ ); on voit clairement que la raie de résonance s'élargit et se déplace vers les champs faibles lorsqu'on augmente l'intensité de la décharge, ou encore la densité des métastables [donc le paramètre $1 / T$ de (3.8) et (3.9)]. Ce résultat correspond bien à l'augmentation de la fréquence de précession prévue par la théorie.

Pour une intensité de décharge donnée, nous avons répété la mesure du déplacement et de la largeur de la résonance à diverses valeurs du champ magnétique.

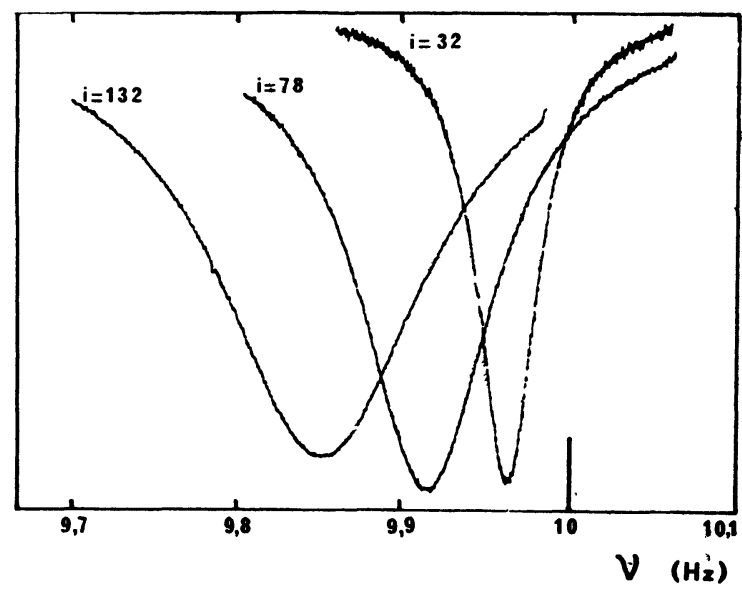

Fig. 8. - Résonance magnétique nucléaire de ${ }^{3} \mathrm{He}$ pour des intensités $i$ croissantes de la décharge. On observe un élargissement et un déplacement de la résonance. La position de la résonance, extrapolée à $i=0$, correspond à la valeur $v_{\mathrm{f}}=10 \mathrm{~Hz}$ de la fréquence de résonance nucléaire.

Les résultats expérimentaux sont représentés par les points de la figure 9. Le déplacement et la demilargeur sont exprimés en hertz. Le champ magnétique est mesuré par la fréquence de résonance $v_{\mathrm{f}}$. Les courbes de la figure 9 sont théoriques; elles sont tracées en utilisant les formules (3.8) et (3.9), où $\tau$ a été déterminé d'après les résultats du paragraphe 5.1 précédent et $T$ ajusté empiriquement. L'accord entre les courbes théoriques et les points expérimentaux est satisfaisant, compte tenu des incertitudes sur $\tau$.

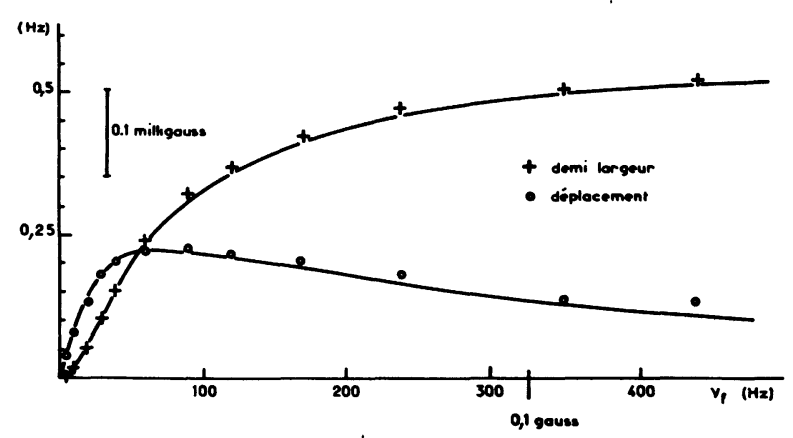

Fig. 9. - Variations du déplacement et de la demi-largeur de la résonance magnétique nucléaire de ${ }^{3} \mathrm{He}$ en fonction du champ statique, pour une intensité fixée de la décharge. Les points représentent les résultats expérimentaux. Les courbes en traits pleins sont théoriques (on a ajusté l'échelle verticale sur la valeur expérimentale de la largeur de raie en champ fort).

\section{Références}

[1] Dupont-Roc, J., Leduc, M. et Lalö̈, F., J. Physique 34 (1973) 961.

[2] Donszelmann, A., Thèse, Amsterdam (1970); Physica 56 (1971) 138

[3] Dupont-Roc, J., Thèse, Paris (1972); C.R. Hebd. Séan. Acad. Sci. B 273 (1971) 45 et 283
[4] Leduc, M., Thèse, Paris (1972).

[5] Dupont-Roc, J., Leduc, M. et Lalö̈, F., Phys. Rev. Lett. 27 (1971) 467.

[6] Colegrove, F. D., Schearer, L. D. et Walters, G. K., Phys. Rev. 132 (1963) 2561.

[7] Rosner, S. D., Pipkin, F. M., Phys. Rev. A 5 (1972) 1901. 